\title{
Wohlfahrtsmärkte in Deutschland - Eine voraussetzungsvolle Form der Sozialpolitik
}

In den letzten zwei Jahrzehnten sind Marktmechanismen in verschiedenen Bereichen des deutschen Wohlfahrtsstaats eingerichtet und ausgebaut worden. Nutzer sollen nun in sogenannten Wohlfahrtsmärkten zwischen den Produkten konkurrierender Anbieter wählen. Allerdings stellt sich die Frage, ob damit sozialpolitische Ziele erreicht werden können bzw. unter welchen Voraussetzungen durch Märkte eine sozialpolitisch befriedigende Versorgung sichergestellt werden kann. Beides hängt, so die hier vertretene These, wesentlich von den Nutzern selbst und ihren Fähigkeiten ab. Es muss sichergestellt werden, dass sie über die materiellen Ressourcen verfügen, die eine Marktteilnahme ermöglichen; dass sie genügend Informationen besitzen und auf einem transparenten Markt agieren; und dass sie schließlich auch fähig sind, die richtige Entscheidung zu treffen. Es ist keineswegs klar, ob diese Voraussetzungen in den deutschen Wohlfahrtsmärkten auch erfüllt werden. Häufig scheint eine weitergehende Regulation vonnöten. ${ }^{1}$

\section{1 \\ Einleitung}

Reformen im deutschen Sozialstaat zielten seit den 1990er Jahren unter anderem auf mehr Markt und Wettbewerb. Dadurch sollten eine effizientere Versorgung sichergestellt und Kosten für die öffentlichen Haushalte gesenkt, aber auch den Nutzern durch Wahlfreiheit eine bessere Versorgung ermöglicht werden. In Wohlfahrtsmärkten können Nachfrager nach sozialen Gütern und Dienstleistungen zwischen verschiedenen, konkurrierenden Anbietern wählen. Prominente Beispiele sind neben dem Pflegedienstleistungsmarkt die Märkte für ambulante und stationäre Gesundheitsdienstleistungen, der Markt für Versicherungsprodukte der öffentlich-rechtlichen Krankenkassen, der durch freie Kassenwahl und die Ermöglichung von Wahltarifen entstanden ist, der Markt für staatlich geförderte Altersvorsorgeprodukte kommerzieller Anbieter („Riesterrente“) und im Politikfeld Beschäftigung die Märkte für Vermittlung und Weiterbildung. ${ }^{2}$

Diese Wohlfahrtsmärkte weisen eine explizite sozialpolitische Zielsetzung und deswegen auch Regulierung auf. Auch wenn die Verbesserung der Position der Nutzer nur ein Motiv unter anderen für die Einrichtung der Märkte gewesen ist, sollen sie der Versorgung mit bestimmten, sozialpolitisch relevanten Gütern und Dienstleistungen dienen. Allerdings ist die Erreichung dieses sozialpolitischen Ziels durchaus voraussetzungsvoll, da es neben dem Funktionieren der Märkte auch vom Verhalten und den Ressourcen der Nutzer abhängt, ob eine angemessene Versorgung sichergestellt wird. Dieser Artikel zielt auf die Klärung der Voraussetzungen, unter denen Märkte die sozialpolitische Zielsetzung einer angemessenen Versorgung erreichen können. Dabei präsentiere ich zugleich Hinweise darauf, ob diese Voraussetzungen in den deutschen Wohlfahrtsmärkten erfüllt sind. ${ }^{3}$

In der deutschen Forschungsliteratur existiert bereits eine Reihe von Arbeiten, die Wohlfahrtsmärkte - auch politikfeldübergreifend - behandeln, darunter mehrere, die neue Anforderungen an die Nutzer und Verbraucherfragen in den Mittelpunkt rücken (zuletzt Lamping 2009; siehe auch Nullmeier 2002a). Allerdings besteht eine Forschungslücke, weil bisher die Erfolgsvoraussetzungen der Märkte, insbesondere die Voraussetzungen auf der Nachfrageseite, nicht systematisch analysiert wurden. Eine Diskussion der Wohlfahrtsmärkte und insbesondere der Voraussetzungen ihres Nutzens erscheint auch deshalb notwendig, weil die christlich-liberale Koalition in ihrem Koalitionsvertrag ein klares Bekenntnis zur staatlich geförderten privaten Altersvorsorge ablegte, weitere Wahlmöglichkeiten in der Krankenversicherung schaffen will und im Bereich Pflege die soziale Pflegeversicherung durch ein kapitalgedecktes, verpflichtendes und individualisiertes Element ergänzen möchte (CDU/CSU/FDP 2010, S. 84, 86, 90, 93).

Im Folgenden werden die Erfolgsbedingungen von Wohlfahrtsmärkten herausgearbeitet, wobei ein Schwerpunkt auf die Nachfrageseite, d. h. auf die notwendigen Ressourcen und Fähigkeiten der Nutzer, gelegt wird. Dazu wird zunächst ein Überblick über die deutschen Wohlfahrtsmärkte und die sie behandelnde wissenschaftliche Debatte gegeben und daran anschließend die Bedeutung der Nachfrageseite für den sozialpolitischen Nutzen von Wohlfahrtsmärkten erläutert (Abschnitt 2). Darauf aufbauend werden in drei Schritten die Voraussetzungen für einen sozialpolitischen Erfolg von Wohlfahrtsmärkten diskutiert und mögliche Probleme aufgezeigt. Ich argumentiere, dass Nutzern der Zugang zu Märkten ermöglicht werden muss (Abschnitt 3), diskutiere die in der Literatur zu Wohlfahrtsmärkten häufig geäußerte An-

Ich danke den zwei anonymen Gutachtern und der Redaktion der WSI-Mitteilungen für ihre hilfreichen Anmerkungen.

2 Vermarktlichungstendenzen im Bereich Bildung und Kindererziehung werden in der deutschen Fachdebatte meist ausgeblendet; die Diskussion ist hier nach wie vor durch ihren Fokus auf sozialpolitische Leistungen im engeren Sinn, insbesondere diejenigen, die im Zusammenhang mit den klassischen öffentlich-rechtlichen Sozialversicherungen stehen, dominiert. Die nachfolgenden Überlegungen können zumindest in einigen Aspekten auch auf andere Märkte übertragen werden.

3 Der Artikel zielt nicht auf eine umfassende Bewertung des Erfolgs oder Misserfolgs der deutschen Wohlfahrtsmärkte ab. Die Diskussion und die Präsentation von empirischen Hinweisen sollen mögliche Probleme, Schwachstellen und Regulierungsbedarf aufzeigen helfen.

Florian Blank ist Wissenschaftler im Wirtschafts- und Sozia/wissenschaftlichen Institut (WSI) in der Hans-Böckler-Stiftung. Arbeitsschwerpunkt: Sozialpolitik. e-mail: Florian-Blank@boeckler.de 
sicht, dass Nutzer mehr Informationen brauchen, um eine angemessene Entscheidung in Märkten treffen zu können (Abschnitt 4), und frage anknüpfend an Arbeiten, die das Konstrukt des "homo oeconomicus“ insgesamt angreifen, inwiefern Bürger fähig sind, erfolgreich auf Märkten zu handeln (Abschnitt 5). Bei allen drei Punkten wird jeweils auf die deutschen Wohlfahrtsmärkte Bezug genommen. Schließlich zeige ich auf, welche politischen Handlungsnotwendigkeiten und -möglichkeiten sich aus diesen verschiedenen Aspekten ergeben (Abschnitt 6) und fasse die Ergebnisse der Diskussion zusammen (Abschnitt 7).

\section{2 \\ Wohlfahrtsmärkte - Nachfragesteuerung und Eigenverantwortung}

In der deutschen Sozialpolitik ist seit Mitte der 1990er Jahre eine verstärkte Nutzung von Marktmechanismen zur Erreichung sozialpolitischer Ziele festzustellen. Im Zuge der Einführung der „Riesterrente“ Anfang 2002, die von vielen Beobachtern als ein Bruch mit der bisherigen Rentenpolitik wahrgenommen wurde, haben sogenannte Wohlfahrtsmärkte auch in der deutschen Wohlfahrtsstaatsforschung verstärkte Aufmerksamkeit erfahren. Die deutsche Debatte knüpft dabei an angelsächsische Arbeiten an (Le Grand 1993; Bartlett et al. 1998; Taylor-Gooby 1999), hat aber bald einen eigenen Fokus entwickelt. Hier werden vorwiegend solche Märkte einzeln oder im Überblick - diskutiert,

- die funktional in einem Zusammenhang mit den klassischen Sozialversicherungen und ihren Sicherungszielen stehen, - auf denen die Nutzer (Versicherte, Patienten, Pflegebedürftige etc.) als Nachfrager auftreten, und

- die einer sozialpolitischen Regulierung unterliegen. ${ }^{4}$

Damit sind sowohl Märkte ausgeklammert, auf denen zwar sozialpolitisch relevante Güter gehandelt werden, die aber einer spezifischen Regulierung entbehren, als auch Märkte, die ein Mittel der internen Steuerung von Institutionen sind oder die Beziehungen zwischen Institutionen regeln. Gerade die spezifische Regulierung soll sicherstellen, dass durch die Märkte sozialpolitische Ziele erreicht werden: Sie soll einen bestimmten Konsum ermöglichen oder sogar garantieren. Darum ist auf allen Wohlfahrtsmärkten nicht nur eine Regulierung des Angebots, sondern eine deutliche Steuerung der Nachfrageseite durch Anreize oder eine quasi-öffentliche Nachfrage vorzufinden.

In der deutschen Debatte finden vor allem die folgenden Märkte Beachtung, nicht zuletzt, weil sie Ergebnisse von Reformen der für den deutschen Wohlfahrtsstaat typischen Sozialversicherungssysteme darstellen:

(1) Die Einführung der staatlichen Förderung privater Altersvorsorge („Riesterrente") hat das Augenmerk auf Vermarktlichungsprozesse gelenkt. In diesem Markt bieten kommerzielle Anbieter Vorsorgeprodukte an (u. a. Versicherungen, Bankund Fondssparpläne). Die öffentliche Hand versucht, durch Zulagen und Steuerermäßigungen die Nachfrage der förderberechtigten Bürger zu stimulieren. Durch den privaten Konsum von Vorsorgeprodukten soll das Ziel einer Lebensstandardsicherung im Alter erreicht werden, die durch die gesetzliche Rentenversicherung (GRV) als nicht mehr realisierbar angesehen wird.

(2) Seit den 1990er Jahren wurde ein Wettbewerb zwischen den gesetzlichen Krankenkassen initiiert, der sich lange Zeit über unterschiedliche Beitragssätze, teils auch über andere Faktoren (Qualität von Angebot und Beratung, verschiedene Wahltarife) abspielte. In jüngster Zeit findet der Preiswettbewerb über die kassenabhängigen Zusatzbeiträge der Versicherten statt. Durch die allgemeine Versicherungspflicht (seit 2007) wird die Nachfrage hier durch den Staat hergestellt.

(3) In der ambulanten wie der stationären medizinischen Versorgung kann von einem Markt insofern ausgegangen werden, als dass hier die Anbieter von Dienstleistungen (teils karitative Organisationen, teils öffentliche Institutionen, teils selbstständige Unternehmer oder Konzerne) um Patienten werben, die ihrerseits formal freie Wahl zwischen den Anbietern haben. Der Zugang zu den Gütern und Dienstleistungen wird durch Krankenversicherungen garantiert, wobei erhebliche Unterschiede zwischen gesetzlichen und privaten Krankenversicherungen und teils auch zwischen verschiedenen Tarifmodellen innerhalb eines Systems bestehen.
(4) Im Bereich Pflege wurde mit der Einführung der Pflegeversicherung ein Markt geschaffen, in dem erstmals private und gemeinnützige Anbieter einander gleich gestellt wurden. Pflegebedürftige erhalten durch die (obligatorische) Pflegeversicherung monetäre gestufte Leistungen, die sie bei einem Anbieter ihrer Wahl verwenden können. Allerdings sind die Leistungen nur als ein Zuschuss zu den tatsächlichen Kosten angelegt.

(5) Schließlich sind im Bereich Arbeit und Beschäftigung Gutscheinsysteme entstanden, mit denen Arbeitslose Weiterbildungsund Vermittlungsmaßnahmen von kommerziellen Anbietern nutzen können. Der Zugang zu diesen Gutscheinen ist teils ein Rechtsanspruch, hängt teils aber auch von der Einschätzung eines Sachbearbeiters ab.

Generell werden Märkten einige Vorteile zugeschrieben: Einerseits können Nutzer - so die Annahme - unter den Angeboten das Beste für sie passende auswählen oder den Anbieter zu einem Eingehen auf ihre Bedürfnisse zwingen (Sicherung und Steigerung von Qualität durch Wahlmöglichkeiten; Responsivitäts- und Diversifizierungs-Argument). Andererseits können Nachfrager gerade bei standardisierten Angeboten gelenkt durch den Preis das kostengünstigste Angebot auswählen und damit einen höheren sozialpolitischen Nutzen erzielen (Rationalisierungsargument) (Lamping 2009, S. 45). ${ }^{5}$ Die Schaffung von Marktmechanismen und von Entscheidungsfreiheit ist entsprechend immer auch mit einer (partiellen) Verantwortungsverlagerung verbunden und mit einem höheren Risiko für die Nutzer: Die Wahl zwischen Anbietern und Produkten beinhaltet auch immer die Möglichkeit, eine falsche Entscheidung zu treffen. In der Forschungsliteratur ist wiederholt auf diese gewandelte Rolle der Nutzer und die Anforderungen, die Märkte als Mittel der Sozialpolitik mit sich bringen, hingewiesen

4 Siehe für verschiedene Aspekte der deutschen Debatte Nullmeier 2001; 2002a; Leisering et al. 2002; Bode 2005; 2008; Berner 2009; Blank 2009; Lamping 2009.

5 Die potenziellen Vorzüge von Märkten sollten nicht vergessen machen, dass die genannten Marktmechanismen in Deutschland auch vor dem Hintergrund des Versuchs eingeführt wurden, Kosten zu reduzieren und teilweise auf die Nutzer zu verlagern. Letzteres gilt insbesondere für die "Riesterrente". 
worden; ebenso wird teils auch auf Fragen des Verbraucherschutzes eingegangen (Nullmeier 2001; Ewert 2009; Lamping 2009). Allerdings sind in der Debatte die Voraussetzungen für einen sozialpolitischen Erfolg der Wohlfahrtsmärkte nicht systematisch analysiert worden.

Wohlfahrtsmärkte als Mittel der Sozialpolitik sollten in erster Linie an ihrem sozialpolitischen Nutzen gemessen werden. ${ }^{6}$ Das Ziel, das durch Wohlfahrtsmärkte erreicht werden soll, besteht ganz grundlegend in der adäquaten Versorgung der Bevölkerung mit Gütern und Dienstleistungen. Adäquate Versorgung bedeutet, dass die Nicht-Inanspruchnahme von Gütern und Dienstleistungen nur auf der freien Entscheidung der Nutzer beruht und nicht auf Marktversagen oder mangelnden Ressourcen der Nutzer - und dass durch Märkte nicht bestimmte Nutzergruppen systematisch besser versorgt werden als andere. Dieses Ziel bildet letztlich den Hintergrund für die sozialpolitischen Eingriffe auf der Nachfrageseite der Wohlfahrtsmärkte.

Es ist den Wohlfahrtsmärkten eigen, dass eine angemessene Versorgung außer vom Funktionieren des Marktes vom Verhalten der Nachfrager abhängt, genauer: von der Fähigkeit der Bürger, die richtigen Entscheidungen zu treffen. Damit ergibt sich für die Sozialpolitik ein Dilemma: Die Vorteile, die sich aus der Kombination von Marktmechanismen und sozialpolitischen Zielen ergeben sollen (Responsivität und Effizienz), beruhen auf der Fähigkeit der Nutzer, aus Eigeninteresse die richtigen Entscheidungen zu treffen. Das Dilemma ergibt sich daraus, dass im Unterschied zu anderen Märkten der Nutzen der konkreten Entscheidung zwar vom Einzelnen bemessen werden soll (und dies als Wahlfreiheit auch einen Eigenwert darstellen kann), aber mit ihr zugleich ein politisch relevanter sozialpolitischer Nutzen verbunden wird (selbst wenn dieser individuell und nicht gesamtgesellschaftlich gefasst wird) (Moore 2002, S. 298f.). Es scheint auf der Grundlage dieser Überlegungen sinnvoll, bei einer Prüfung der Voraussetzungen für das Erreichen des sozialpolitischen Ziels die Nachfrageseite in den Vordergrund zu rücken: Unter welchen Umständen können die Nutzer die für ihre Versorgung notwendigen, „richtigen“ Entscheidungen treffen? ${ }^{7}$

Eine erfolgreiche Nutzung - und damit der sozialpolitische Erfolg von Wohl- fahrtsmärkten - setzt dreierlei voraus: Erstens muss den Nutzern der Zugang zu den Wohlfahrtsmärkten materiell möglich sein. Hier geht es um die für eine Marktteilnahme notwendigen Ressourcen und damit auch die institutionellen Rahmenbedingungen, durch die die Nachfrage in Wohlfahrtsmärkten beeinflusst oder geschaffen wird. Zweitens müssen für ein erfolgreiches Handeln der Nutzer die Märkte transparent sein und die für die Transaktionen notwendigen Informationen zur Verfügung stehen. Drittens sollen auf dieser Grundlage die Individuen selbst fähig sein, die richtigen Entscheidungen zu treffen, die ihre soziale Sicherung berühren.

In den folgenden drei Abschnitten werden diese Aspekte behandelt. Dabei werden jeweils auch Ergebnisse der empirischen Forschung angeführt, die auf Schwierigkeiten in den deutschen Wohlfahrtsmärkten hinweisen. Sie liefern keinen absoluten Beweis für Erfolg oder Scheitern der Märkte, sondern deuten auf Risiken und Regulierungsbedarfhin. Die Einbeziehung dieser Forschungsergebnisse erscheint deswegen als angemessen, weil der sozialpolitische Nutzen der Wohlfahrtsmärkte letztlich von der tatsächlich vorhandenen Möglichkeit der erfolgreichen individuellen Nutzung abhängt - die Feststellung von Defiziten, die zu einer suboptimalen Versorgung führen, stellt damit, auch wenn sie nur einzelne Gruppen oder Aspekte betrifft, ein sozialpolitisches Problem dar und verweist auf Handlungsbedarf zumindest im Detail, möglicherweise aber sogar auf tiefer liegende Probleme mit der Nutzung von Marktmechanismen.

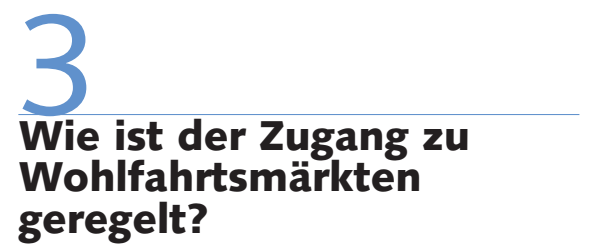

Im vorigen Abschnitt wurde auf die zentrale Rolle der Nutzer und die damit verbundene Regulation der Nachfrage in Wohlfahrtsmärkten hingewiesen. Eine grundlegende Voraussetzung für die Versorgung mit Gütern und Dienstleistungen durch Märkte ist schlicht der Zugang zu diesen Märkten: Eine adäquate Versorgung ist nur dann möglich, wenn alle Betroffenen die Ressourcen haben (und erübrigen können), die zum Erhalt sozialer Güter und Dienstleistungen notwendig sind.
Ein Vergleich der im letzten Abschnitt kurz dargestellten Wohlfahrtsmärkte in Deutschland zeigt deutliche Unterschiede in der jeweiligen sozialpolitischen Regulierung des Zugangs und damit der Schaffung und Förderung der Nachfrage. Diese Unterschiede lassen sich wie folgt systematisieren (vgl. Blank 2009):

(1) Ist die Marktteilnahme freiwillig oder obligatorisch - können also Nutzer selbst über ihre Versorgung entscheiden oder besteht eine andere Regelung? Die deutschen Wohlfahrtsmärkte zeigen hier verschiedene Ausprägungen: Das „Riestern“ ist freiwillig, in der Krankenversicherung herrscht Versicherungspflicht, über die Vergabe von Weiterbildungsgutscheinen entscheiden Sachbearbeiter.

(2) Welche Ressourcen werden den Nutzern gegeben und wie werden sie berechnet? AuBer einem direkten Anspruch gegenüber dem Anbieter (Sachleistungsprinzip im Gesundheitswesen) lassen sich unterschiedliche kalkulierte monetäre Ressourcen (Grundzulagen bei der privaten Alterssicherung) oder Gutscheinsysteme (Vermittlungsgutscheine) finden. Die Gewährung von Ressourcen kann auch an Eigenleistungen oder Vorleistungen geknüpft sein (Riesterförderung nur bei Eigenbeitrag).

(3) Wie hängen Märkte mit anderen Sicherungssystemen zusammen? Werden Märkte als Ergänzung zu weiter existierenden öffentlichen Systemen eingeführt (wie die Gutscheine in der Arbeitsvermittlung oder die „Riesterrente“) oder werden sie in bestehende Systeme implementiert (Wettbewerb zwischen den gesetzlichen Krankenkassen)?

Durch diese Stellschrauben sind unterschiedlich intensive Eingriffe ins Marktgeschehen möglich, die die Nachfrage nach

6 Dies ist allerdings nur ein Blickwinkel zur Evaluation von Wohlfahrtsmärkten. Ebenso kann beispielsweise nach den Voraussetzungen ihrer Effizienz oder explizit nach Gerechtigkeitsaspekten gefragt werden (Le Grand 1993).

7 Die Überlegung, dass der sozialpolitische Nutzen von den individuellen Entscheidungen abhängt, ist der Grund für den Fokus auf die Nachfrageseite in diesem Beitrag. Eine angebotsseitige Regulierung von Märkten - beispielsweise die Regulation von Produkten - ist notwendig und auf den Wohlfahrtsmärkten auch gegeben, sie wird hier aber nur angesprochen, wenn die Entscheidungsmöglichkeiten der einzelnen Nutzer direkt betroffen sind. 
Gütern und Dienstleistungen beeinflussen. Es macht für die Nutzer einen gewaltigen Unterschied, ob sie selbst über den Zutritt entscheiden, hierbei auch noch eigene Ressourcen investieren müssen und daneben die öffentliche Unterstützung auf einen Zuschuss beschränkt bleibt - wie im Falle der Riesterrente. Oder ob die individuelle Marktteilnahme gar nicht zur Debatte steht und eine öffentliche Förderung der Nachfrage dann greift, wenn sie selbst die notwendigen Ressourcen nicht aufbringen können - wie im Falle der Entscheidung zwischen miteinander konkurrierenden gesetzlichen Krankenversicherungen.

Die Frage des Marktzugangs ist ein erster Ansatzpunkt, wenn es darum geht, den sozialpolitischen Nutzen von Märkten und seine Voraussetzungen zu bestimmen. Die materielle Schaffung oder Förderung der Nachfrage ist eine zentrale Möglichkeit, eine adäquate Versorgung durch Märkte sicherzustellen. In den deutschen Wohlfahrtsmärkten gibt es Hinweise darauf, dass mangelnde Ressourcen zu einer mangelhaften Versorgung beitragen können: 2008 wurde die Anpassung und Dynamisierung der Leistungen der Pflegeversicherung mit der Begründung beschlossen, dass sie seit 1995 unverändert geblieben seien und damit einem Wertverfall unterlägen, wodurch Pflegebedürftige zunehmend von der Sozialhilfe unterstützt werden müssten (Deutscher Bundestag 2007, S. 40). Auch Analysen zur „Riesterrente" deuten darauf hin, dass Menschen mit geringem Einkommen relativ seltener sparen (Statistisches Bundesamt 2009, S. 9; Geyer/Steiner 2009; Coppola/Reil-Held 2009). Sozialpolitisch sind die Ergebnisse des Marktgeschehens hier zu kritisieren, da der als notwendig angesehene Konsum nicht stattfinden kann.

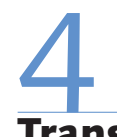

\section{Transparente Märkte und informierte Markt- teilnehmer}

Als grundlegendes Problem im Zusammenhang mit Wohlfahrtsmärkten wird vielfach die Ausstattung der Nachfrager mit Informationen genannt, die diese für eine zweckmäßige Entscheidung benötigen (wobei diese Entscheidung auch die Frage nach dem Markteintritt generell betreffen kann). In der Vergangenheit ist häufiger darauf hingewiesen worden, dass neue Wohlfahrtsmärkte aufseiten der Nutzer auch eines neuen "Marktwissens“ (Nullmeier 2002b, S. 108) bedürfen. Aber inwieweit können Laien durch Informationen soweit gebildet werden, dass sie einem hoch spezialisierten Gegenüber gewachsen sind? Muss nicht in vielen Bereichen - etwa in der Beziehung zwischen Patienten und Ärzten - von einer permanenten Informationsasymmetrie ausgegangen werden? Forderungen nach mehr Information werden allerdings durch Beobachtungen unterstützt, die etwa auf eine verstärkte Nachfrage nach Informationen im Gesundheitssektor hinweisen (Marstedt 2003), aber auch durch Untersuchungen, die für den Gesundheitssektor einen Mangel an - aus Sicht der Nutzer - relevanter Information belegen (Geraedts 2008, S. 44) oder die ein bestimmtes Verhalten direkt mit Informationsdefiziten verknüpfen. So können fehlende oder falsche Informationen über die vermeintlichen negativen Folgen eines Kassenwechsels die Wechselbereitschaft von Versicherten mindern, eine Erhöhung der Wechselbereitschaft könnte also durch bessere Information der Versicherten erreicht werden (Greß et al. 2008, S. 88). Auch im Bereich Pflege ist eine hohe Wertschätzung von Beratung mit einer geringen Nutzung von Beratungsstellen und einem geringen Wissen um diese verbunden (Bauer 2008, S. 241ff.) und ebenso wird für die Alterssicherung eine Stärkung der finanziellen Bildung gefordert (Leinert 2004, S. 29).

Mit diesen Forderungen nach mehr Informationen korrespondiert die Kritik an mangelnder Transparenz in den Wohlfahrtsmärkten. Diese Kritik bezieht sich sowohl auf einzelne Informationsmöglichkeiten wie die Pflegetransparenz-Vereinbarungen (Möller/Zieres 2010) als auch ganze Märkte wie die „Black Box“ Krankenhaussektor (Streuf et al. 2007), den Markt für Arbeitsvermittlung (Bernhard et al. 2008, S. 24) und den Markt für „Riesterrenten“, dessen fehlende Transparenz auch durch unabhängige Information nur tendenziell behoben werden könne (Hagen/Reisch 2010, S. 10).

Empirische Untersuchungen, wie etwa zum Programm „Altersvorsorge macht Schule ${ }^{\text {"8 }}$ zeigen auch, wie schwer es ist, alle betroffenen Gruppen mit Informationen zu erreichen. Das von verschiedenen Institutionen und Verbänden unterstützte Projekt soll Bürgerinnen und Bürger zu informierten Entscheidungen hinsichtlich ihrer Altersvorsorge befähigen. Die Kursteilnehmer zeichnen sich unter anderem durch überdurchschnittliche Bildung aus, Angestellte sind gegenüber anderen Gruppen überrepräsentiert und Kursteilnehmer haben häufig bereits eine zusätzliche Altersvorsorge (Frommert 2008, S. 341). Für die Nutzung von Gesundheitsinformationen aus dem Internet stellt Marstedt (2003, S. 135) eine ähnliche Tendenz fest: „Gerade die älteren und niedrig Qualifizierten, die häufiger krank sind und deswegen den größten Bedarf an zusätzlichen Informationen haben müssten, nutzen dieses Medium am seltensten“. Die Konsequenz aus diesen Beobachtungen könnten zielgruppenspezifische Programme zur Informations- und Kompetenzvermittlung sein, bei denen auch die Kosten der Informationsbeschaffung Berücksichtigung finden. Allerdings bleiben damit grundlegende Probleme ungelöst, die mit dem Bild des „mündigen Verbrauchers“ insgesamt und nicht mit bestimmten Nutzergruppen verbunden sind.

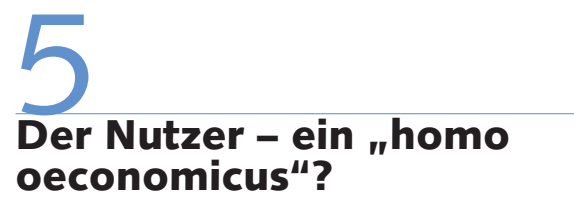

Rufe nach Information und Verbraucherbildung sowie nach mehr Transparenz sind mit dem Bild eines mündigen Konsumenten verbunden, der fähig und willens ist, die richtigen Entscheidungen zu treffen - wenn er denn nur die richtigen Informationen hat. Dass dieses Bild auch der Politik der gegenwärtigen Bundesregierung zugrunde liegt, zeigt ihre Antwort auf eine parlamentarische Anfrage zur Riesterrente: Die Bundesregierung setzt „[...] in ihrer Verbraucherpolitik grundsätzlich auf die Stärkung des Verbrauchers [...]. Leitbild ist dabei der gut informierte mündige Verbraucher und ein Markt, auf dem mit überschaubarem Aufwand ein Vergleich von Angeboten möglich ist" (Deutscher Bundestag 2010, S. 2). Gegen das Bild des mündigen Verbrauchers lassen sich allerdings grundlegende Vorbehalte äußern, insofern er deutliche Spuren des „homo oeconomicus" der neoklassischen Wirtschaftstheorie trägt.

8 http://www.altersvorsorge-macht-schule.de 
Diese Vorbehalte sind von Sozialwissenschaftlern schon früher im Zusammenhang mit Wohlfahrtsmärkten vorgebracht worden (Taylor-Gooby 1999; Bode 2005). Einen wichtigen Ansatzpunkt liefern zudem Einsichten der „behavioral economics“. Diese experimentell ausgerichtete Forschungsrichtung legt nahe, dass Verbraucher einer ganzen Reihe von Beschränkungen unterliegen, die nicht nur in Einzelfällen zu irrationalem Verhalten führen: Sie neigen unter anderem zu Aufschiebeverhalten, bewerten Informationen entsprechend ihrer Präsentation, können Informationen nur in einem begrenzten Umfang verarbeiten oder verzetteln sich in Details, legen die Entscheidungsmaßstäbe in Abhängigkeit von früheren (und begrenzten) Erfahrungen und Informationen fest, neigen zur Selbstüberschätzung und zur Kontrollillusion, bewerten Kosten und Nutzen in Gegenwart und Zukunft unterschiedlich, haben Probleme, die akkumulierten Kosten von einzelnen Entscheidungen zu bewerten und können ihr eigenes zukünftiges Verhalten nur schwer voraussagen (Ratner et al. 2008; Oehler/ Reisch 2008). Diese Erkenntnisse sind zum Teil direkt auf Probleme der Altersvorsorge und des Verbraucherschutzes in der Alterssicherung bezogen worden. ${ }^{9}$ Sie lassen sich aber auch auf andere Bereiche der sozialen Sicherung anwenden. Schließlich ist bei den meisten im Bereich der Sozialpolitik gehandelten Produkten selbst für rational handelnde Individuen der individuelle Nutzen nur schwer zu bemessen, einerseits, weil er weit in der Zukunft liegt oder nur in bestimmten, individuell zufälligen Situationen gemessen werden kann (Versicherungen), oder aber, weil seine genaue Beurteilung beträchtliches Fachwissen voraussetzt (Gesundheitsdienstleistungen und -produkte). ${ }^{10}$

Dass diese Bedenken nicht bloß theoretischer Natur sind, belegen Analysen zu den verschiedenen Märkten im deutschen Sozialsystem. Auf dem Markt für Arbeitsvermittlung hält man es für möglich „[...] dass gerade Personen mit Vermittlungshemmnissen mit der Selbstauswahl eines privaten Vermittlers überfordert sind. Dies könnte zum einen dazu führen, dass diese Personen ihren Vermittlungsgutschein weniger häufig einlösen. Zum anderen könnte es aber auch eine Erklärung dafür sein, dass dieser Personenkreis, selbst wenn er den Gutschein einlöst, tendenziell weniger profitiert, weil ein weniger gut passender oder gar qualitativ schlecht arbeitender Vermittlungsdienstleister ausgewählt wird“ (Bernhard et al. 2008, S. 24). Dieselben Autoren berichten außerdem mit Blick auf Weiterbildungsgutscheine, dass Arbeitsvermittler der Arbeitsagenturen angäben, arbeitsmarktferne Arbeitslose seien häufig mit der Auswahl einer Maßnahme überfordert (Bernhard et al. 2008, S. 30; siehe auch Kruppe 2008, S. 29).

Im Bereich Gesundheit ist grundsätzlich zu fragen, wie viel Wissen überhaupt realistischerweise aufseiten der Versicherten und Patienten verlangt werden kann. So wirft Marstedt (2003, S. 135) die Frage nach der Qualität des „,medizinischen Laienwissens" von Patienten auf und gibt zu bedenken, dass dieses Wissen auch verwirrend und desorientierend wirken könne (siehe auch Gerlinger 2009, S. 34). Zum Wechselverhalten von Krankenversicherten ist festzuhalten, dass trotz einer hohen Preissensitivität der Versicherten und einer hohen Konsumentenmobilität gegen einen Wechsel nicht nur die geringen Unterschiede zwischen den Krankenkassen, sondern auch die Gewohnheit sprechen (Greß 2006, S. 51) - dieser Faktor ist gerade kein rationales Entscheidungsmoment.

Im Falle der „Riesterrente“ zeigt sich, dass Sparer teilweise auf staatliche Zulagen verzichten, indem sie beispielsweise Anträge auf Zulagen oder Sonderausgabenabzug nicht stellen. 2003 wurden mindestens 13,4 Mio. €, 6,5 \% der staatlichen Förderung nicht abgerufen (Kriete-Dodds 2008, S. 62). Grundsätzlich stellt sich zudem die Frage, auf welcher Grundlage Entscheidungen in diesem Markt getroffen werden können. Angesichts der Vielzahl der Produkte - bis zum 30. November 2010 wurden laut Bundeszentralamt für Steuern über 5.000 „Riester-“ und „Rürup-“ Produkte zertifiziert ${ }^{11}-$, die in vielerlei Hinsicht nicht standardisiert sind und in ihrer Menge auch nicht durch Produkttests vollständig bewertet werden können, kann hier eine optimale, informierte Entscheidung kaum gefällt werden. Hagen/Reisch (2010, S. 13) kommen entsprechend zu dem Urteil, „[...] dass der Markt der privaten Altersvorsorge allein aus strukturellen Gründen zu Funktionsstörungen neigt: Dies ist auf Qualitätsunsicherheit und systematisch irrationales Verhalten der Verbraucher zurückzuführen“.

Die in den letzten drei Abschnitten vorgestellten Überlegungen zeigen, dass die Erreichung sozialpolitischer Ziele durch Marktmechanismen höchst voraussetzungsvoll ist: Die Verbraucher müssen Zugang zu den Wohlfahrtsmärkten haben, über die notwendigen Informationen verfügen und außerdem zu einem angemessenen Verhalten auf den Märkten fähig sein. Während die erste Bedingung, der Zugang zu Märkten - den politischen Willen vorausgesetzt -, relativ leicht zu erfüllen ist, hängen die anderen Bedingungen von den Marktstrukturen und den Fähigkeiten der Individuen ab. Sind die Voraussetzungen nicht erfüllt, muss das als sozialpolitisch unbefriedigend angesehen werden, solange das Ziel der angemessenen Versorgung mit Gütern und Dienstleistungen besteht - eine weitergehende Regulierung der Märkte erscheint dann als notwendig. Dies kann allerdings mit einem der politischen Motive für die Einführung von Wohlfahrtsmärkten, nämlich der Senkung von Kosten, kollidieren.

\section{Ist Regulierung eine Lösung?}

Angesichts der genannten Voraussetzungen und der durch sie bedingten potenziellen Probleme stellt sich die Frage, ob und wie Märkte reguliert werden können, um sozialpolitischen Zielen zu genügen. Sozialpolitisch ist zunächst die Frage nach der grundsätzlichen Marktteilnahme relevant - verfügen alle Bürgerinnen und Bürger über die notwendigen materiellen Ressourcen oder gibt es einen Rechtsanspruch auf Zugang zu Gütern und Dienstleistungen? Hier steht nicht das Wollen, sondern das Können im Vordergrund (Lamping 2009, S. 59). Soll nun den Bürgern in manchen Märkten die freie Entscheidung zur Marktteilnahme überlassen bleiben? Lässt sich eine Art der Regulierung finden, die für die allermeisten Bürger die Möglichkeit einer erfolgreichen Teilnahme erhöht?

9 S. hierzu Hagen/Reisch 2010; Hippe 2007, S. 494ff.; Thaler/Sunstein 2009, S. 113ff., $151 \mathrm{ff}$. Zu den Grenzen der "finanziellen Allgemeinbildung" s. Tiffe 2009.

10 Es handelt sich hier um Vertrauens- und Kontraktgüter (Hagen/Reisch 2010, S. 5).

11 Dabei ist allerdings zu berücksichtigen, dass ein Teil der Zertifikate wieder zurückgegeben wurde. http://www.bzst.de/cae/servlet/contentblob/ 163272/publicationFile/3666/Lister_aller_ erteilten_Zertifikate_XLS.xls 
(1) Eine Pflicht zur Marktteilnahme in Versicherungsmärkten, verbunden mit einer Bereitstellung der notwendigen Ressourcen, entbindet von der Entscheidung, ob eine individuelle Marktteilnahme jetzt oder später notwendig ist. Dies bedeutet noch keine Garantie einer erfolgreichen Entscheidung, entlastet die Bürger aber. Eine mildere Form wäre eine obligatorische Marktteilnahme mit Optout-Regelung, wie sie beispielsweise von Vertretern des „libertarian paternalism“ im Zusammenhang mit Betriebsrenten vorgeschlagen wird (Thaler/Sunstein 2009, S. 118ff.). Allerdings muss dabei bedacht werden, dass durch Opting-out sozialen Sicherungssystemen notwendige Ressourcen abhandenkommen können und der Umfang der öffentlichen Verantwortung gegenüber denjenigen zu klären ist, die sich gegen eine Marktteilnahme entscheiden.

(2) Der direkte Zugang zu Sach- und Dienstleistungen konkurrierender Anbieter als ein Recht kann ebenfalls dazu beitragen, diese eher in Anspruch zu nehmen, auch wenn daraus andere Probleme wie eine extensive Nutzung eines solchen Rechts folgen können (aber nicht müssen).

Was das Problem der Entscheidungsgrundlagen und des tatsächlichen Verhaltens der Marktteilnehmer angeht, müssen neben der in der Literatur oft vorgebrachten und oben bereits dokumentierten Forderung nach mehr Information - die trotz ihrer Grenzen nicht per se zurückgewiesen werden sollte - weitere politische Lösungen gefunden werden. Sozialpolitik rückt hier in die Nähe von Verbraucherpolitik (Nullmeier 2001, S. 93ff.).

(1) Evtl. kann in vielen Bereichen eine öffentliche oder unabhängige (zivilgesellschaftliche oder kommerzielle) Beratung im Sinne eines milden Führens installiert werden, vielleicht sogar als obligatorische Beratung hinsichtlich der Marktteilnahme und der Produktwahl; dies könnte nicht nur als Verbraucherinformation oder -beratung, sondern als "Verbraucherbeeinflussung“ (Lamping 2009 S. 59) gesehen werden, wobei die Kompetenzen und Beschränkungen unterschiedlicher Bevölkerungsgruppen dringend mitberücksichtigt werden sollten.

(2) Notwendig erscheint die kritische Prüfung des Nutzens bereits vorhandener Informationen. So sehen einige beispielsweise das Vorhandensein von Gütesiegeln und Zertifikaten „als eine der am meisten überschätzen Eigenschaften bei der Wahl eines Krankenhauses“ (Streuf et al. 2007, S. 118).

Eine weitergehende Regulierung kann direkt an der Angebotsseite der Märkte ansetzen. Dies fügt sich insofern in die bisherigen Überlegungen zu den Voraussetzungen auf der Nachfrageseite, als dass die folgenden Vorschläge in allererster Linie auf eine Komplexitätsreduktion aus Sicht der Nutzer abzielen:

(1) Hinsichtlich des Angebots sind qualitätssichernde Maßnahmen bereits üblich. Weitergehende Regulierungen wie eine Standardisierung von Produkten (z.B. Versicherungsverträgen) und Kosten könnten die Informationsüberflutung der Verbraucher bekämpfen helfen.

(2) Gegebenenfalls muss auch über die Schaffung von öffentlichen Anbietern in bisher privat bedienten Bereichen oder eine Konzentration von Anbietern nachgedacht werden. Eine weitere Möglichkeit ist die Setzung eines vertrauenswürdigen Anbieters als Standardoption bei verpflichtender Marktteilnahme. Allerdings zeigt das Beispiel der schwedischen Alterssicherungspolitik, dass der bei der „Prämienrente“ - einem obligatorischen kapitalgedeckten Versicherungszweig - als Standardauswahl vorgegebene, öffentlich verwaltete Rentenfonds im Laufe der Jahre schlechtere Ergebnisse erzielen, als die kommerzielle Konkurrenz (Köhler 2010, S. 111).

Auf der Nachfrageseite ließe sich über eine Bündelung der Nachfrage oder eine Vermittlung durch Intermediäre nachdenken, etwa vertrauenswürdige zivilgesellschaftliche Institutionen, die entweder selbst als Nachfrager auftreten oder zwischen Angebot und Nachfrage vermitteln (Gruppentarife). Diese Rolle kann allerdings mit hohen politischen Kosten verbunden sein, da bei einer suboptimalen Leistung der Anbieter die Intermediäre verantwortlich gemacht werden könnten.

Politische Regulierung kann zudem auch bei den Arbeitsbedingungen ansetzen, die auf der Anbieterseite gegeben sind, um die Verbraucher vor Schlechtleistungen zu bewahren - dies zielt insbesondere auf Fälle, in denen eine Exit-Option des Verbrauchers nur schwer kurzfristig zu realisieren ist. Das betrifft nicht nur per- sonenbezogene Dienstleistungen, sondern auch Beratungsdienstleistungen im Finanzsektor. Das Ver.di-Vorstandsmitglied Uwe Foullong wies beispielsweise in einem Interview auf die Probleme hin, die durch Zielvorgaben an Bankangestellte entstehen (Schröder/Jahberg 2010); hier geraten die Aufgaben „Beratung“ und „Verkauf“ in Konflikt.

Vor dem Hintergrund der Pläne der schwarz-gelben Koalition sollte die Sozialpolitik schließlich reflektieren, auf wie vielen Märkten Bürger realistischerweise handeln können - die genannten Wohlfahrtsmärkte machen letztlich nur einen Teil der Dienstleistungs- und Gütermärkte aus, auf denen sie aktiv sind. Gleichzeitig sind die Wohlfahrtsmärkte aber von immenser Bedeutung für ihr individuelles aktuelles und zukünftiges - Wohlergehen. Entsprechend ist zu klären, inwieweit eine öffentliche Verantwortung gegenüber denjenigen besteht, die im Markt scheitern.

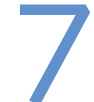 \\ Fazit}

Sozialpolitik hat es grundsätzlich mit der Deckung bestimmter Bedarfe zu tun. Die politische Festlegung, welche Bedürfnisse befriedigt werden sollen, lässt sicherlich unterschiedliche Wege der Leistungserbringung zu. Die Forderung nach Wahlfreiheit ergibt vor dem Hintergrund eines voll ausgebauten und leistungsfähigen Wohlfahrtsstaates durchaus Sinn - vor allem wenn er, wie in anderen Ländern, durch fehlende Anbietervielfalt gekennzeichnet ist. Auch in Bereichen, wo der sozialpolitische Erfolg mit von der Qualität zwischenmenschlicher Beziehungen abhängt, erscheint es angemessen, Kunden und Patienten eine gewisse Wahlfreiheit zu lassen (beispielsweise freie Arztwahl oder Wahl eines Pflegedienstes). Es erscheint bei allen Hinweisen auf mögliche Probleme mit Märkten auch notwendig, daran zu erinnern, dass auch nicht den Marktgesetzen unterworfene öffentliche Einrichtungen Versicherte, Patienten, Hilfebedürftige nicht immer so behandeln wie diese es wünschen oder wie es angemessen erscheint. Verbraucherschutz und Verbraucherrechte können damit auch in einem öffentlichen Sozialsystem notwendig sein.

Es wurde dargelegt, dass die Verfolgung sozialpolitischer Ziele durch Märkte äußerst voraussetzungsvoll ist. Es deutet 
vieles darauf hin, dass unter den gegenwärtigen Bedingungen den Verbrauchern viel zugetraut, aber auch viel zugemutet wird. Einige Probleme lassen sich durch einen verbesserten Marktzugang bzw. eine bessere Ressourcenverteilung und durch Informationsvermittlung sicherlich mildern, allerdings stellt sich weiterhin die Frage, ob und inwiefern Menschen auf Märkten - gerade mit Blick auf sozialpolitische Güter - erfolgreich sein können. Die Beobachtungen und Argumente der „behavioral economics“ legen nahe, dass Nutzer teils einer expliziten Anleitung bedürfen, die auch über auf den ,homo oeconomicus" gemünzte ökonomische Anreize hinausgeht. Da die sozialpolitische
Regulierung von Märkten aber bestimmten Versorgungszielen dienen soll, muss immer geprüft werden, welcher Nutzengewinn durch Wahlfreiheit angesichts der Beschränkungen, denen Marktteilnehmer unterliegen, überhaupt erzielt werden kann und - grundsätzlicher - ob Sozialpolitik auf das Mittel des Marktes zurückgreifen sollte. Letztendlich kann eine verbesserte Unterstützung der Nutzer, also die Regulierung von Märkten, helfen, das sozialpolitische Ziel einer angemessenen Versorgung zu realisieren. Allerdings sind mit solchen regulativen Maßnahmen wiederum Kosten verbunden. Hier stellt sich abstrakt die Frage nach dem Wert von Wahlfreiheit in der Sozialpolitik, konkret aber das Pro- blem, dass Marktmechanismen auch zur Kostendämpfung eingesetzt werden sollen. Die Alternative zu mehr Wahlfreiheit angesichts der hohen Wahrscheinlichkeit, dass Märkte nicht für alle Teilnehmer ein optimales Ergebnis produzieren, ist ein starker öffentlicher Beitrag zur Distribution von Gütern und Dienstleistungen. Dies insbesondere, weil Fehlentscheidungen von (potenziellen) Marktteilnehmern nicht nur zu gesellschaftlich möglicherweise noch vertretbaren individuellen Nutzenverlusten führen können, sondern in der Summe auch sozialpolitisch relevante Problemlagen herbeiführen können, die dann doch wieder von der Allgemeinheit gelöst werden müssen.

\section{LITERATUR}

Bartlett, W./Roberts, J. A./Le Grand, J. (1998) (Hrsg.): A Revolution in Social Policy. Quasi-market Reforms in the 1990s, Bristol Bauer, U. (2008): Die Zukunft der Pflege: Qualitäts- und Strukturfragen aus Nutzersicht, in: Böcken., J./Braun, B./Amhof, R. (Hrsg.): Gesundheitsmonitor 2008. Gesundheitsversorgung und Gestaltungsoptionen aus der Perspektive der Bevölkerung, Gütersloh, S. 231-249

Berner, F. (2009): Der hybride Sozialstaat. Die Neuordnung von öffentlich und privat in der sozialen Sicherung, Frankfurt a.M./New York Bernhard, S./Hohmeyer, K./Jozwiak, E./Koch, S./Kruppe, T./Stephan, G./Wolff, J. (2008): Aktive Arbeitsmarktpolitik in Deutschland und ihre Wirkungen, IAB-Forschungsbericht 2, Nürnberg

Blank, F. (2009): When „Choice" and "Choice" Are not the Same: Institutional Frameworks of Choice in the German Welfare System, in: Social Policy \& Administration 43 (6), S. 585-600

Bode, I. (2005): Einbettung und Kontingenz. Wohlfahrtsmärkte und ihre Effekte im Spiegel der neueren Wirtschaftssoziologie, in: Zeitschrift für Soziologie 57 (4), S. 250-269

Bode, I. (2008): The Culture of Welfare Markets. The International Recasting of Pension and Care Systems, New York/London

Christlich Demokratische Union Deutschlands (CDU), Christlich-Soziale Union in Bayern (CSU), Freie Demokratische Partei (FDP) (2010): Wachstum. Bildung. Zusammenhalt. Der Koalitionsvertrag zwischen CDU, CSU und FDP, 17. Legislaturperiode, Berlin

Coppola, M./Reil-Held, A. (2009): Dynamik der Riesterrente: Ergebnisse aus Save 2003 bis 2008, MEA Discussion Paper 195, Mannheim Deutscher Bundestag (2007): Gesetzentwurf der Bundesregierung. Entwurf eines Gesetzes zur strukturellen Weiterentwicklung der Pflegeversicherung (Pflege-Weiterentwicklungsgesetz), Bundestag-Drucksache $16 / 7439$, Berlin

Deutscher Bundestag (2010): Antwort der Bundesregierung auf die Kleine Anfrage der Abgeordneten Matthias W. Birkwald, Caren Lay, Klaus Ernst, weiterer Abgeordneter und der Fraktion DIE LINKE, Drucksache 17/501, Berlin [Bundestag-Drucksache 17/677]
Ewert, B. (2009): Economization and Marketization in the German Healthcare System: How Do Users Respond?, in: German Policy Studies 1, S. 21-44

Frommert, D. (2008): Zur Evaluation der Bildungskampagne "Altersvorsorge macht Schule“, in: Deutsche Rentenversicherung 3, S. 327342

Geraedts, M. (2008): Informationsbedarf und Informationssuchverhalten bei der Arztsuche, in: Böcken, J./Braun, B./Amhof, R. (Hrsg.): Gesundheitsmonitor 2008. Gesundheitsversorgung und Gestaltungsoptionen aus der Perspektive der Bevölkerung, Gütersloh, S. 29-47 Gerlinger, R. (2009): Wettbewerb und Patientenorientierung in der gesetzlichen Krankenversicherung, in: Böckmann, R. (Hrsg.): Gesundheitsversorgung zwischen Solidarität und Wettbewerb, Wiesbaden, S. 19-41 Geyer, J./Steiner, V. (2009): Zahl der Riester-Renten steigt sprunghaft - aber Geringverdiener halten sich noch zurück, in: DIW Wochenbericht 32 , S. 534-541

Greß, S. (2006): Regulierter Wettbewerb in der sozialen Krankenversicherung: Ein Dreiländervergleich, in: Internationale Revue für Soziale Sicherheit 59 (3), S. 33-57

Greß, S./Höppner, K./Marstedt, G./Rothgang, H./Tramm, M./Wasem, J. (2008): Kassenwechsel als Mechanismus zur Durchsetzung von Versicherteninteressen, in: Braun, B./Greß, S./Rothgang, H./Wasem, J. (Hrsg.): Einfluss nehmen oder aussteigen. Theorie und Praxis von Kassenwechsel und Selbstverwaltung in der Gesetzlichen Krankenversicherung, Berlin, S. 19-89

Hagen, C./Reisch, L. A. (2010): Riesterrente: Politik ohne Marktbeobachtung, in: DIW Wochenbericht 8, S. 2-14

Hippe, T. (2007): Märkte, Wohlfahrtsstaaten oder Wohlfahrtsmärkte? Typen der Regulierung kapitalgedeckter Altersvorsorge im Vergleich, in: Deutsche Rentenversicherung 8-9, S. 492-504

Köhler, P. A. (2010): Die neue Alterssicherung Schwedens in der globalen Finanzkrise - bedingt Krisenfest?, in: Deutsche Rentenversicherung 1 , S. 102-118 
Kriete-Dodds, S. (2008): Staatliche Förderung der Riesterrente für das Jahr 2003, in: Wirtschaft und Statistik 1, S. 60-68

Kruppe, T. (2008): Selektivität bei der Einlösung von Bildungsgutscheinen, IAB-Discussion Paper 17, Nürnberg

Lamping, W. (2009): Verbraucherkompetenz und Verbraucherschutz auf Wohlfahrtsmärkten: Neue Herausforderungen an eine sozialpolitische Verbraucherpolitik, in: Vierteljahreshefte zur Wirtschaftsforschung 78 (3), S. 44-62

Le Grand, J. (1993): Ein Wandel in der Verwendung von Policy-Instrumenten: Quasi-Märkte und Gesundheitspolitik, in: Héritier, A. (Hrsg.): Policy-Analyse, Kritik und Neuorientierung, Opladen, S. 225-244 Leinert, J. (2004): Finanzieller Analphabetismus in Deutschland. Schlechte Voraussetzungen für eigenverantwortliche Vorsorge, in: Gesundheits- und Sozialpolitik 58 (3-4), S. 24-30

Leisering, L./Davy, U./Berner, F./Schwarze, U./Blömeke, P. (2002):

Vom produzierenden zum regulierenden Wohlfahrtsstaat. Eine international vergleichende und interdisziplinäre Studie des Wandels der Alterssicherung in Europa, Regina Arbeitspapier 2, Bielefeld Marstedt, G. (2003): Auf der Suche nach gesundheitlicher Information und Beratung: Befunde zum Wandel der Patientenrolle, in: Böcken, J./Braun, B./ Schnee, M. (Hrsg.): Gesundheitsmonitor 2003. Die ambulante Versorgung aus Sicht der Bevölkerung und Ärzteschaft, Gütersloh, S. 117-135

Möller, J./Zieres, G. (2010): Zur Transparenz der Qualität von Pflegeeinrichtungen, in: Gesundheitsökonomie \& Qualitätsmangement 15 (1), S. 39-44

Moore, M. H. (2002): Privatizing Public Management, in: Donahue, J. D./Nye Jr., J. S. (Hrsg): Market-Based Governance. Supply Side, Demand Side, Upside, And Downside, Washington D.C., S. 296-322 Nullmeier, F. (2001): Sozialpolitik als marktregulative Politik, in: Olk, T./Evers, A./Heinze, R. G. (Hrsg.): Baustelle Sozialstaat - Umbauten und veränderte Grundrisse, Jahrestagung der Sektion "Sozialpolitik“ der Deutschen Gesellschaft für Soziologie, 4./5. Mai, Wiesbaden, S. 77-99
Nullmeier, F. (2002a): Demokratischer Wohlfahrtsstaat und das neue Marktwissen, in: Heinrich-Böll-Stiftung (Hrsg.): Gut zu Wissen - Links zur Wissensgesellschaft, Münster, S. 97-111

Nullmeier, F. (2002b): Auf dem Weg zu Wohlfahrtsmärkten?, in: Süß, W. (Hrsg.): Deutschland in den neunziger Jahren, Opladen, S. 269-281

Oehler, A./Reisch L. A. (2008): Behavioral Economics - eine neue Grundlage für Verbraucherpolitik? Eine Studie im Auftrag des Verbraucherzentrale Bundesverbandes, Berlin

Ratner, R. K./Soman, D./Zauberman, G./Ariely, D./Carmon, Z./Keller, P. A./Kim, B. K./Lin, F/Malkoc, S./Small, D. A./Wertenbroch, K. (2008): How behavioral decision research can enhance consumer welfare: From freedom of choice to paternalistic intervention, in: Marketing Letters 19 (3), S. 383-397

Schröder M./Jahberg H. (2010): „Bankberater erinnern an Drückerkolonnen“, http://www.zeit.de/wirtschaft/2010-01/verdi-kritikbankberater (20.01.2010)

Statistisches Bundesamt (2009): Staatliche Förderung der Riesterrente 2004, Wiesbaden

Streuf, R./Maciejek, S./Kleinfeld. A./Blumenstock, G./Reiland, M./ Selbmann, H. K. (2007): Informationsbedarf und Informationsquellen bei der Wahl eines Krankenhauses, in: Gesundheitsökonomie und Qualitätsmanagement 12, S. 113-120

Taylor-Gooby, P. (1999): Markets and Motives. Trust and Egoism in Welfare Markets, in: Journal of Social Policy, 28 (1), S. 97-114

Tiffe, A. (2009): Verbraucherpolitik im Bereich der Finanzdienstleistungen muss mehr sein als Bereitstellung von Information, in: DIW Wochenbericht 8, S. 110-124

Thaler, R. H./Sunstein, C. R. (2009): Nudge. Improving Desicions About Health, Walth and Happiness, London 